\title{
LE BOULANGISME ET LA COMMUNE
}

Rechercher des liens de filiation possibles entre la Commune et le mouvement boulangiste présente au premier abord un caractère paradoxal. Car le général Boulanger, officier de carrière, a combattu les Communards dans les rangs de l'armée de Versailles. Et les adversaires de Boulanger dans le mouvement ouvrier et socialiste n'ont pas manqué de rappeler ce souvenir. C'est ce que fait notamment l'ouvrier Avronsart, dans une interview de Boulanger dont l'authenticité fut d'ailleurs contestée. ${ }^{1}$ Plus tard, lorsque Boulanger pose sa candidature à l'élection partielle de la Seine, se constitue contre lui un groupe qui s'intitule précisément "la Commune» et qui présente la candidature de Protot. Tout serait à citer de la proclamation de ce groupe, ${ }^{2}$ dont nous nous bornerons à extraire ce raccourci historique quelque peu hardi :

"Robespierre tue la Commune et fait Bonaparte; Cavaignac tue la Commune et refait Bonaparte; $M$. Thiers tue la Commune et voilà Boulanger."

Mais cette tentative tourne court; la candidature Protot est bientôt retirée; $;^{3}$ c'est la démonstration qu'en 1889 il n'est plus possible de mobiliser les masses autour du seul souvenir de la Commune. Cependant les Boulangistes socialisants ont jugé nécessaire de répondre à l'argument personnel. Cette réponse incombait au premier chef à Rochefort, qui avait bâti pour une large part sa popularité dans le public parisien sur le fait qu'il figurait parmi les victimes de la répression versaillaise. Rochefort répond essentiellement que Boulanger n'était alors qu'un soldat astreint à la discipline militaire, donc irresponsable, ${ }^{4}$ et que d'ailleurs il n'a pas laissé de traces dans les

1 In: Paris, numéro du 7 mai 1888, cité par J. Néré, Le Boulangisme et la Presse, Paris 1964, pp. 103-104.

2 Voir le texte intégral dans La Justice, 31 décembre 1888.

3 La Justice, 13 janvier 1889.

4 L'Intransigeant, 31 mai 1888. Le même argument est repris par Séverine dans Le Cri du Peuple, 7 janvier 1888, cité par J. Néré, Le Boulangisme et la Presse, pp. 99-101. 
souvenirs amers des Communards proscrits. Mais c'est là une constatation purement négative. Elle ne suffit évidemment pas à expliquer la triomphale élection de Boulanger comme député de la Seine le 27 janvier 1889, ni le fait, peut-être plus significatif encore, que les députés boulangistes élus en province se présentent presque tous à Paris aux élections générales de $1889,{ }^{1}$ et que Paris - plus précisément même les quartiers périphériques - et la banlieue envoient à la nouvelle Chambre une grande partie de la représentation boulangiste. Paris a-t-il donc radicalement changé entre 1871 et 1889 ? Ou bien certaines idées susceptibles d'entraîner les masses parisiennes peuvent-elles se retrouver à la fois dans la Commune et dans le mouvement boulangiste?

Deux grands thèmes se présentent alors à l'esprit: le nationalisme et ce qu'on peut appeler - en un sens large et peut-être vague - le socialisme. Mais les prendre pour base conduirait à refaire toute l'analyse historique du Boulangisme. Aussi bien l'histoire du mouvement socialiste est déjà riche entre 1871 et 1889 , bien des choses ont évolué, et, comme nous l'avons montré ailleurs, ${ }^{2}$ l'attitude des socialistes organisés vis-à-vis du Boulangisme a dépendu dans une large mesure de leurs positions vis-à-vis des possibilistes, ce qui nous entraîne assez loin de la Commune. Notre propos ici sera beaucoup plus limité: c'est des phénomènes spécifiquement parisiens que nous nous occuperons.

Aux élections générales de 1885, Paris est une forteresse des radicaux, ${ }^{3}$ qui constituent à ce moment l'extrême-gauche de la Chambre des Députés. Cette prépondérance est si nette, que l'élection partielle du 2 mai 1886 est disputée uniquement entre deux candidats qui se réclament également de l'extrême-gauche, Gaulier et Ernest Roche. Plus tard, à une autre élection partielle en mai 1887, le radical Mesureur était élu sans concurrent. Voilà qui répond à notre première question: non, Paris n'a pas radicalement changé d'orientation politique entre la période de la Commune et celle du Boulangisme. Le Conseil Municipal de Paris, à la suite des élections de mai 1887, se compose de 45 aradicaux autonomistes», 3 républicains indépendants, 10 opportunistes, 9 possibilistes, 2 blanquistes, 11 réactionnaires. ${ }^{4}$ C'est sur la signification de cette épithète, "groupe de l'autonomie communale", que nous voudrions nous arrêter quelques instants.

Les lois de 1882 et 1884 ont rendu aux conseils municipaux le droit d'élire leurs maires et adjoints, et la publicité de leurs séances, tout en les laissant assujettis à une tutelle administrative assez stricte. Mais

1 Le Radical, 29 août 1889.

2 Voir un résumé de notre thèse dans Le Boulangisme et la Presse, pp. 98-109.

3 L'Extrême Gauche compte dans la Seine 27 députés sur 37 sièges, selon la liste publiée par Le Radical, 13 février 1886.

4 Selon les listes publiées par Le Radical, 16 mai 1887. 
Paris demeure en dehors du droit commun: il ne possède pas de maire élu, et une grande partie des attributions administratives y sont confiées à un Préfet de Police, fonctionnaire nommé par le gouvernement, dont l'autorité exceptionnelle vient s'ajouter aux prérogatives normales du Préfet de la Seine. Cette méfiance témoignée à l'égard de Paris par la majorité provinciale du Parlement provient sans nul doute dans une large mesure des réactions suscitées par la Commune. Et nous voici au cœur du sujet.

Le conflit permanent entre le Conseil Municipal élu et les deux Préfets nommés crée dans la vie administrative parisienne une situation intenable; ${ }^{1}$ et les conseillers municipaux de Paris devenus députés s'efforcent d'obtenir une réforme dont le trait essentiel serait l'institution d'une mairie de Paris. Durant la législature 1881-1885 la proposition n'a pas abouti; mais en 1886 les chances paraissent meilleures, car désormais aucun ministère nettement républicain ne paraît pouvoir subsister sans l'appui des radicaux $;^{2}$ ceux-ci d'ailleurs ont appris des opportunistes, dans une certaine mesure, les avantages de la prudence tactique, et ils mettent surtout en avant des arguments de logique et d'efficacité administrative. ${ }^{3}$ Au contraire, leurs adversaires placent le problème sur le terrain de la politique générale; ils craignent essentiellement qu'une municipalité parisienne émancipée devienne un Etat dans l'Etat; et c'est évidemment les souvenirs historiques, exprimés ou non, qui dominent le débat. Durant tout le XIXe siècle, ce sont les révolutions parisiennes qui ont imposé à la France ses changements de régime politique. La Commune de Paris - elle portait déjà ce titre - a exercé une forte pression, durant la grande Révolution, sur la Législative et sur la Convention. Et l'on soupçonne la Commune de 1871 d'avoir voulu imiter cet exemple. Les craintes, bruyamment exprimées, que le Conseil Municipal de Paris sorte de son rôle strict d'administration locale, et cherche à peser sur la politique nationale, sont-elles dénuées de tout fondement? Sigismond Lacroix, signataire de la proposition de réforme municipale déposée à la Chambre, et qui comme tel s'applique à lui donner l'allure la plus anodine possible, écrit luimême:

«... la mairie de Paris a toujours coïncidé avec la République triomphante, et c'est la République en décadence qui a toujours préparé les voies à la monarchie par la suppression de la mairie de Paris. $1^{4}$

1 Voir notamment La Justice, 14 juin 1886.

2 J. Néré, Le Boulangisme et la Presse, p. 49.

3 Voir Le Radical, 12 mai 1886, et La Justice, 3 juillet 1886.

4 Le Radical, 16 mai 1886. 
Et plus tard, Rochefort va encore plus loin:

"On doit reconnaître que le Conseil Municipal de Paris est devenu peu à peu notre sauvegarde contre les faiblesses gouvernementales et les compromissions de la Chambre... Il est même probable que le Parlement ne s'obstine à dénier à la capitale son autonomie que par cette haine de ceux qui ne font rien contre ceux qui font quelque chose ....11

Que le Conseil Municipal de Paris se distingue par son activité et la hardiesse de ses initiatives, on pourrait en donner bien des exemples. C'est ainsi qu'il tient à honneur d'être à l'avant-garde du progrès social. Il s'efforce notamment d'introduire le salaire minimum et la limitation de la durée du travail dans les travaux publics qui relèvent de sa compétence. ${ }^{2}$

Bien des faits peuvent être également présentés comme des signes d'hostilité du Parlement à l'égard de Paris. Le projet d'établissement de la mairie de Paris n'aboutit pas - il n'a pas encore abouti aujourd'hui. Il en résulte une profonde désillusion à l'égard des députés radicaux dont on ne comprend guère que dans la nouvelle Chambre ils ne sont toujours pas, malgré leurs effectifs renforcés, en mesure d'imposer leur politique. Or, comme nous nous sommes efforcé de le montrer ailleurs, cette désillusion, la coupure et l'incompréhension croissantes entre les élus et les militants de base du radicalisme, sont à l'origine de l'état d'esprit boulangiste. Ajoutons que ceux des électeurs parisiens dont l'attitude n'est pas déterminée par les questions d'administration ou de politique pure, vont avoir bientôt d'autres griefs. Paris est l'un des premiers centres français à être atteint par la crise économique, qui y sévit cruellement dès 1884. Pour y faire face, le Conseil Municipal envisage un programme de grands travaux, qui doit être financé par un emprunt. En même temps, l'attention parisienne se concentre sur un projet de chemin de fer métropolitain, qui devrait être réalisé pour 1'Exposition Universelle de 1889. Or - à la suite de tractations trop complexes pour être résumées ici - le projet de Métropolitain n'aboutit pas, et le Parlement rejette l'autorisation à la Ville de Paris de lancer un emprunt. L'échec du Métropolitain comme celui de l'emprunt, est présenté comme un "vote contre Paris», et on s'en prend spécialement à cette occasion à l'attitude de certains radicaux de province, ${ }^{\mathbf{3}}$ avec

1 L'Intransigeant, 9 mai 1887.

2 Voir notamment à ce sujet la série des articles de $\mathrm{Ch}$. Longuet dans La Justice, de 1886 à 1887.

${ }^{3}$ Le Radical, 23 juillet 1887; voir aussi L'Intransigeant, 24 octobre 1886, et La Justice, 31 mars 1886 . 
une violence de ton significative. L'hostilité entre province et Paris est donc encore à l'état aigu.

Or ce mécontentement parisien vient coïncider dans le temps avec une vive agitation de l'extrême-gauche dans le domaine de la politique générale. A la suite de l'Affaire Schnæbelé, le général Boulanger est écarté du Ministère de la Guerre, et il se constitue un cabinet Rouvier que les radicaux combattent et qui ne peut donc subsister que par la complaisance de la droite. Il en résulte une levée de boucliers pour la défense de la République ... et de Boulanger, contre Rouvier. ${ }^{1}$ Le Conseil Municipal de Paris joue sa partie dans ce concert; le 27 juillet 1887, adoptant une proposition d'Edouard Vaillant, il invite «tous les conseils municipaux républicains» à envoyer à l'Hôtel de Ville des délégués pour un congrès préparatoire, afin d'organiser la réunion à Paris d'un congrès annuel des communes, «à l'effet de concerter leur action pour la revendication de leurs libertés, des libertés et des droits du peuple et pour la défense de la République». ${ }^{2}$ Cette délibération est annulée par l'autorité de tutelle, ce qui amène Rochefort à écrire: "Il n'y a pas encore bien longtemps, on voyait partout la main de l'Internationale. Aujourd'hui, cette main est devenue celle du général Boulanger. Le Père Grévy a probablement cru entrevoir cette terrible dextre dans la décision prise par le Conseil municipal de Paris, le 27 juillet dernier, et tendant à réunir en Congrès les représentants de tous les Conseils municipaux de France. Aussi s'est-il empressé, au mépris de toutes les lois existantes, d'annuler cette délibération.... ${ }^{3} \mathrm{~A}$ la fin de l'année, le Conseil joue également son rôle dans la crise présidentielle: il envoie son bureau auprès du Congrès, à Versailles, pour mettre en garde le Parlement contre l'éventuelle élection de Ferry. ${ }^{4}$ Or la lutte contre Ferry sera le principal ciment, au niveau des passions populaires, de la coalition boulangiste. ${ }^{5}$

On retrouve donc bien, dans les années qui précèdent l'aventure boulangiste, certains éléments qui ont constitué l'état d'esprit de la Commune, ou qui trouvent leur origine dans l'épisode historique de la Commune. Mais ces élements vont-ils s'incorporer dans le mouvement boulangiste proprement dit une fois que celui-ci a pris toute son originalité? La démonstration directe est difficile à faire. Car le Boulangisme, d'une façon générale, est la résultante assez vague et informe d'une série de mécontentements et de ressentiments dont certains au moins étaient précis. Et la position des cadres politiques à son égard n'est

1 Voir J. Néré, Le Boulangisme et la Presse, pp. 54-55.

2 L'Intransigeant, 29 juillet 1887.

3 L'Intransigeant, 27 août 1887.

4 L'Intransigeant, 4 décembre 1887.

5 Voir J. Néré, Le Boulangisme et la Presse, pp. 60-62. 
souvent pas représentative des sentiments de la masse, comme on le vit notamment lors de l'élection parisienne du 27 janvier 1889. C'est pourquoi il n'est pas très important de remarquer que la majorité du Conseil Municipal de Paris, et notamment de ses membres «autonomistes», se prononça contre Boulanger dès l'entrée officielle de celui-ci dans la bataille politique. ${ }^{1}$ Plus significatives, parce que se situant au niveau des militants de base, sont les prises de position de la "Fédération des groupes républicains socialistes de la Seine», qui sera en 1889 l'organisation la plus utilisée par le Boulangisme parisien. En juillet 1888, elle publie une déclaration ${ }^{2}$ où il est dit notamment:

«[La Fédération] a toujours demandé et elle ne cessera de réclamer la révision immédiate de la Constitution, par une assemblée constituante, dans le sens républicain-socialiste [...]. Elle demande à éliminer de l'organisation politique les rouages non seulement inutiles, mais nuisibles, tels que le Sénat et la Présidence de la République. La Fédération veut aussi que dans le pacte constitutionnel soient inscrites la décentralisation et l'autonomie communale. Sur ce point surtout elle ne capitulera jamais, parce que c'est de là qu'il faut attendre le grand affranchissement."

Les points rappelés ici appartiennent au programme traditionnel des radicaux, mais attention à la date. L'été 1888 , c'est le moment où gouverne pour la première fois un Président du Conseil radical, Charles Floquet. Mais c'est aussi le moment où les radicaux s'avèrent incapables d'appliquer leur programme politique de toujours, et où les partisans les plus ardents et les plus déterminés de la révision constitutionnelle - les «révisionnistes» - ne voient d'autre solution que de passer au Boulangisme. Il n'est pas sans importance de constater que les révisionnistes parisiens font une large place dans leurs revendications à l'autonomie communale, avec ses résonances politiques les plus profondes. C'est à partir de la Fédération que se développent les comités "républicains socialistes révisionnistes» ou "républicains nationaux révisionnistes" d'arrondissement ou de quartier qui assureront au cours de l'année 1889 l'implantation locale du Boulangisme à Paris. Or, si les autorités centrales du mouvement boulangiste évitent soigneusement de publier un programme précis, qui leur aliénerait automatiquement une partie de leurs appuis, les comités locaux sont loin d'observer toujours la même discrétion. Beaucoup adoptent des programmes qui nous ont été conservés. Celui du "comité républicain so-

1 La Justice, 24 avril 1888.

2 Texte intégral dans L'Homme Libre, 17 juillet 1888. 
cialiste révisionniste du IX ${ }^{\mathrm{e}}$ arrondissement", ${ }^{1}$ par exemple, comporte:

(Dans l'ordre politique, suppression de la Présidence de la République et du Sénat, autonomie communale, séparation de l'Eglise et de l'Etat, service militaire de 3 ans égal pour tous ..."

Et dans une réunion du Comité républicain révisionniste du VIe arrondissement, ${ }^{2}$ tenue le 10 janvier 1889 , l'un des orateurs rappelle: «Les députés élus n'ont jamais exécuté leur programme. Malgré les vœux du Conseil Municipal, l'autonomie communale est restée lettre morte, de même que l'exécution du Métropolitain et la suppression des octrois..."

On nous signale des remous dans le Comité républicain révisionniste du XII ${ }^{\mathrm{e}}$ arrondissement ${ }^{3}$ à propos de la candidature aux élections générales de 1889 du Communard Elie May, qui sera d'ailleurs un des élus boulangistes de Paris. L'un des membres de ce comité, Brèche "s'estattiré une sorte de réprobation de la plupart [de ses collègues] par suite de l'horreur insurmontable qu'il montre pour tout ce qui a touché de près ou de loin à la Commune de 1871 . Il a eu à essuyer déjà une foule d'objections à ce sujet..." Le Comité républicain national révisionniste socialiste du XVIII ${ }^{e}$ arrondissement ${ }^{4}$ défraie la chronique en diffusant un programme imprimé comportant une assemblée élue pour deux ans avec mandat impératif; le remplacement des ministres par des fonctionnaires; la suppression de la présidence de la République; le referendum; une décentralisation très poussée; et l'article 11 précise:

«Finances-Budget national voté par l'Assemblée et réparti entre les communes au prorata de leur richesse et de leur population La commune détermine la nature des impôts, le mode de perception et choisit les agents de recouvrement - Répartition de la dette publique entre les régions qui auront à opérer le service des intérêts et de l'amortissement..."

Il paraît difficile de pousser l'autonomie communale plus loin, et l'on conçoit que le Comité National boulangiste, embarrassé par cette profession de foi, ait cherché à l'étouffer. Pourtant le programme du XVIII est accepté par le Comité central républicain socialiste révisionniste du XIX ${ }^{\mathbf{e}}$ arrondissement. ${ }^{5}$ Et il n'est pas inutile de rappeler que c'est dans une circonscription du XVIIIe que Boulanger lui-même se

1 Archives de la Préfecture de Police, Organisations boulangistes, B/a 1465.

2 A.P.P., B/a 1465.

8 A.P.P., B/a 1466.

4 A.P.P., B/a 1468.

5 A.P.P., B/a 1469. 
présenta aux élections générales de septembre 1889 - pour y obtenir d'ailleurs une majorité massive.

Tout cela ne constitue évidemment que des indications fragmentaires, mais il ne faut guère espérer autre chose lorsqu'on étudie le mouvement boulangiste. Et elles suffisent à faire apparaître la persistance de tendances et de sentiments communalistes; à expliquer aussi, autrement que par des considérations purement personnelles, la présence dans le mouvement boulangiste d'anciens Communards. 